\title{
LIGHT CALCULATIONS
}

AND MEASUREMENTS 
P H I L I P T E C H N I C A L L I B R A R Y

\title{
LIGHT CALCULATIONS AND MEASUREMENTS
}

\author{
An introduction to the system of \\ quantities and units in light-technology \\ and to photometry
}

H.A.E. KEITZ

SECOND REVISED EDITION 
(C) N.V. Philips' Gloeilampenfabrieken, Eindhoven (The Netherlands), 1971

Softcover reprint of the hardcover 2nd edition 1971

All rights reserved. No part of this publication may be reproduced or transmitted, in any form or by any means, without permission.

\author{
Published by \\ MACMILLAN AND CO LTD \\ London and Basingstoke \\ Associated companies in New York, Toronto, Melbourne, Dublin, Johannesburg and Madras \\ ISBN 978-1-349-00014-2 ISBN 978-1-349-00012-8 (eBook) \\ DOI 10.1007/978-1-349-00012-8
}

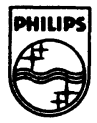

\title{
PHILIPS
}

Trademarks of N.V. Philips' Gloeilampenfabrieken

No representation or warranty is given that the matter treated in this book is free from patent rights; nothing herein should be interpreted as granting, by implication or otherwise, a licence under any patent rights 


\section{FOREWORD}

Since the first edition of this book appeared 14 years ago, it has been widely used in lighting practice and as a textbook, and appears to have received an enthusiastic response from users. It was therefore possible to leave the form and content of the book basically unchanged in this second edition.

A new section on Dourgnon and Fleury's quasi-central projection has been added to the chapter on the representation of light distributions. A section on the determination of the reflection properties of road surfaces in the chapter on reflection, absorption and transmission has completely been revised. Finally, a new section on the special measures to be taken when measuring the properties of gas-discharge lamps, with or without fittings, appears in the chapter on the measurement of luminous flux. The absorption of colour filters has also been dealt with in somewhat greater detail than in the first edition.

Measuring techniques have become much more mechanized and automated of recent years; electronics, and in particular digital techniques, are widely used in this connection. Such methods, which are also widely used for lighting measurements, belong to the field of electronics rather than that of lighting proper. They have therefore not been discussed in detail in this book, which is mainly concerned with basic photometric methods.

The author has made grateful use of the remarks and suggestions received from many readers since the appearance of the first edition. The whole text and the illustrations have also been carefully revised, and it is hoped that this second edition will also be of service to its readers in the practical and educational fields.

Thanks are due to all who have helped in the production of this book, and in particular to Prof. Dr. H. W. Bodmann of the University of Karlsruhe (until recently attached to the Philips' Lighting Laboratory in Aachen) who read the entire revised second edition through, and to whom many improvements in the text are due. 


\section{O N T E N T S}

Page

PART I

Chapter I

\section{LIGHT CALCULATIONS}

Introduction

I-1. The nature of light. . . . . . . . . . . . 1

2. Light as a wave-phenomenon . . . . . . . . 3

3. Polarisation. . . . . . . . . . . . . . . . 7

4. Photometry; the photometric system of Lambert . 8

5. The development of lighting engineering . . . . . 9

6. Subjects dealt with in this book. . . . . . . . 16

Chapter II

Solid Angle

II-1. Solid angle, steradian . . . . . . . . . . . . . . 18

2. Some special solid angles .. . . . . . . . . 19

3. Significance of the conception "solid angle" in illuminating engineering . . . . . . . . . . 21

4. Small solid angles . . . . . . . . . . 21

5. Table of solid angles . . . . . . . . . . . 24

Chapter III Luminous Flux, Luminous Intensity, Quantity

of Light

III-1. Luminous flux; luminous intensity . . . . . . . 25

2. Units of luminous intensity and luminous flux . . 28

3. Formulae giving the relationship between luminous intensity and luminous flux . . . . . . . . . 32

4. Horizontal, spherical luminous intensity. . . . . . 33

5. Luminous efficiency . . . . . . . . . . . . . . . 35

6. Quantity of light, lumen-second, lumen-hour . . 35

Chapter IV Light Distribution, Rousseau and Zonal Luminous Flux Diagrams

IV-1. Light distribution, the Rousseau diagram . . . . . 39

2. Construction of the Rousseau diagram . . . . . . 45

3. Derivation of the Rousseau diagram with the aid of the infinitesimal calculus . . . . . . . . . 45

4. Determination of the efficiency of a lighting fitting from the Rousseau diagram . . . . . . . . . 
5. The zonal luminous flux diagram . . . . . . . 48

6. The area enclosed within the luminous intensity curve is not a measure of the luminous flux emitted 50

7. Average luminous intensity calculated from the Rousseau diagram . . . . . . . . . . 51

8. Russel angles. . . . . . . . . . . . . . . . 51

9. The long-base Rousseau diagram for narrow beams of light . . . . . . . . . . . . . . . 53

10. Detailed example: determination of a 1000 -lm curve for a symmetrical lighting fitting. . . . . . . . 54

11. Classification of lighting fittings according to their light distibution . . . . . . . . . . 58

\section{Chapter V Methods of Representing Light Distribution}

V-1. Luminous intensity table; polar and rectangular light distribution diagrams . . . . . . . . . 60

2. The isocandela diagram . . . . . . . . . 60

3. Spherical co-ordinates . . . . . . . . . 62

4. Sinusoidal projection . . . . . . . . . . 62

5. Transformation of spherical co-ordinates . . . . 64

6. The azimuthal projection . . . . . . . . . 67

7. Comparison of the sinusoidal and the azimuthal projections . . . . . . . . . . . . 70

8. Quasi central projection . . . . . . . . . 71

9. The application of preferred numbers in isocandela diagrams . . . . . . . . . . . . . . . . 74

10. Examples of isocandela diagrams of a fitting . . .76

VI-1. Illumination; foot-candle; lux . . . . . . . . . 78

2. The inverse square law . . . . . . . . . 79

3. Illumination with oblique incidence. . . . . . 81

4. Horizontal and vertical illumination . . . . . . 85

5. Other units of illumination . . . . . . . 86

Chapter VII Illumination Calculations and Diagrams

VII-1. Introduction . . . . . . . . . . . . . . . . . . 88

2. The lumen method; coefficient of utilization . . . 88

3. The point-by-point method . . . . . . . . 90 
4. Illumination diagrams . . . . . . . . . . . . . 92

5. The rectangular illumination diagram . . . . . . 93

6. The solid of illumination. . . . . . . . . . 995

7. The plane isolux diagram . . . . . . . . . 95

8. The polar isolux diagram . . . . . . . . . . . 96

9. Determination of the luminous flux from the plane isolux diagram . . . . . . . . . . . . . 99

10. The isolux diagram in quasi central projection . . 100

\section{Chapter VIII Luminance and Luminous Emittance}

VIII-1. Luminance; the stilb, candelas per sq. in. . . . . . 104

2. Lambert's law . . . . . . . . . . . . . 105

3. The luminous flux of uniform diffusers . . . . 106

4. Luminous flux of a uniformly diffuse cylinder. . . 108

5. Luminous emittance . . . . . . . . . . . . 111

6. Emittance and luminance of uniformly diffusing surfaces . . . . . . . . . . . . 112

7. Units of luminance, based on the emittance of uniform diffusers ........... . 112

IX-1. Luminous intensity of non-point sources . . . . . 115

2. The inverse square law in another form . . . . . 120

3. Universal formula for the illumination produced by uniformly diffuse circular light sources . . . . . 121

4. Alternative derivations of the formula $E=\pi L \sin ^{2} \Theta 124$

5. Luminance of light beams . . . . . . . . . . . . 128

6. Comparison between the illumination values obtained from equations $E=L \omega$ and $E=\pi L \sin ^{2} \Theta 128$

7. Some special applications of the formula $E=\pi L \sin ^{2} \Theta=I / a^{2}$. . . . . . . . . . . . . . 130

8. Illumination produced by a uniformly diffuse circular light source in planes parallel to the source . 131

9. Illumination produced by linear light sources . . . 136

10. Comparison of illumination values of linear light sources as obtained by exact calculation with those obtained by means of the inverse square law . . . 140

11. Other kinds of non-point source . . . . . . . . . 143

12. The significance of the foregoing considerations as applied to practical forms of light source . . . . 143 
Chapter X Reflection, Absorption, Transmission

$\mathrm{X}-1$. Reflection, absorption, transmission. . . . . . 146

2. Regular reflection . . . . . . . . . . . . . 147

3. Reflection factors of non-conductive materials . . 149

4. Reflection factor of conductive materials . . . . . 152

5. Diffuse reflection . . . . . . . . . . . 152

6. Uniform diffuse reflection . . . . . . . . . . 157

7. Luminance factor . . . . . . . . . . . . . . . . 158

8. The luminance factor as a constant of the material 162

9. Gloss . . . . . . . . . . . . . . . . . 167

10. Reflection from road surfaces . . . . . . . . 168

11. Transmission ............ . . 174

12. Density . . . . . . . . . . . . . 176

13. Diffusion factor. . . . . . . . . . . . . 178

14. Mụltiple reflection . . . . . . . . . . 180

15. Absorption . . . . . . . . . . . . . . . . 183

16. Absorption of coloured filters . . . . . . . 187

17. Colour of reflected light. . . . . . . . . 188

Chapter XI Froperties of Optical Systems

XI-1. Introduction . . . . . . . . . . . . . 190

2. Relationship between luminance and refractive index; Abbe's law . . . . . . . . . . . . . . . . . 191

3. Luminance of images formed by lenses and mirrors, and of the lenses and mirrors themselves . . . . 196

4. Optical systems as light sources; the exit pupil of optical systems . . . . . . . . . . . . . . . 200

5. Determination of the exit pupil of an optical system; entrance pupil . . . . . . . . . . . . 203

6. Object at the focus of a lens; angle of divergence . 205

7. The exit pupil of lens systems; vignetting . . . . 207

8. The luminous flux of optical systems. Aperture of lens and mirror systems. . . . . . . . . 212

9. Some remarks on mirror systems . . . . . . . . 214

10. Drum lenses . . . . . . . . . . . . . 214

11. Cylindrical mirrors . . . . . . . . . . 216

12. Facetted mirrors . . . . . . . . . . . . . 217

13. Optical systems for the projection of images . . . 218

14. Diascopic projection. . . . . . . . . . 219 
15. Slide projection. . . . . . . . . . . 221

16. Standard-film projection . . . . . . . . . 225

17. Sub-standard film projection . . . . . . . . 227

18. The luminous flux emitted by projection systems .228

19. Episcopic projection. . . . . . . . . 230

Chapter XII The Photometric Measuring-units System

XII-1. Introduction . . . . . . . . . . . . 233

2. The Luminance criterion . . . . . . . . . . . . 234

3. Luminance of coloured light . . . . . . . . . 236

4. The relative luminous efficiency of radiation . . . 239

5. The summation law. . . . . . . . . . . . . . 242

6. Definitions of photometric quantities and units based on $V_{\lambda}$. . . . . . . . . . . . . . . . . . 243

7. Vision at high and low luminance levels; Purkinje effect . . . . . . . . . . . . . . . . . . . . 248

8. Equivalent luminance . . . . . . . . . . . 251

9. Minimum perceptible luminance difference and sensitivity to luminance difference . . . . . . . 254

XIII-1. Introduction. Principles of visual and physical photometry . . . . . . . . . . . . . . . . . . 263

2. Standard light sources . . . . . . . . . . 264

3. Simultaneous and substitution methods . . . . . 268

4. Some practical hints . . . . . . . . . . . 269

Chapter XIV Visual Photometry and Photometers

XIV-1. Principle of the visual photometer . . . . . . . 273

2. Forms of photometer field . . . . . . . . . . 276

3. Methods used in photometers for obtaining the variation of luminance . . . . . . . . . . 282

4. Photometer bench. Some examples of visual photometers . . . . . . . . . . . . . . . 286 
5. Homochromatic and heterochromatic photometry . 291

6. Choice of observer . . . . . . . . . . . 292

Chapter XV Fhysical Photometers and Photometry

$\mathrm{XV}-1$. Principles of physical photometers and photometry 295

2. Photo-emissive cells . . . . . . . . . . . . 296

3. Photo-voltaic cells . . . . . . . . . . . . 302

4. Bolometers and thermo-couples . . . . . . . 313

5. Photographic photometry .. . . . . . . . 314

6. Physical photometers for heterochromatic photometry 315

Chapter XVI Measurement of Luminous Intensity

XVI-1. Principle of luminous intensity measurement . . 321

2. The photometer bench . . . . . . . . . . 322

3. Apparatus for measurement of light distribution . . 324

4. Measurements on projectors . . . . . . . . . . 329

5. Photometer with Maxwellian view . . . . . . 332

Chapter XVII Measurement of Luminous Flux, Quantity of Light and Luminous Emittance

XVII-1. The Ulbricht sphere photometer . . . . . . . 335

2. Illumination of the sphere window in the "ideal" integrating photometer . . . . . . . 336

3. Illumination of the window of a non-ideal integrating photometer. . . . . . . . . . . . . . . 338

4. Measures to be taken with non-ideal integrating photometers to approximate to the ideal sphere 340

5. The measurement of the luminous flux of fluorescent lamps and the appropriate fittings .... . . 351

6. Determination of luminous flux from the light distribution or from an isolux diagram . . . . . 355

7. Measurement of quantity of light . . . . . . 355

8. Measurement of luminous emittance . . . . . 357

Chapter XVIII Measurement of Illumination

XVIII-1. Introduction . . . . . . . . . . . . 359

2. Measurement by means of laboratory photometers 360

3. Visual illumination photometers . . . . . . 360 
4. Physical illumination photometers . . . . . . 362

5. Calibration of illumination photometers. . . . 366

Chapter XIX Measurement of Luminance

XIX-1. Direct visual measurement of luminance . . . . . 367

2. Visual and physical luminance measurements obtained from measurement of illumination . . . . . 372

3. Measurement of luminance distribution . . . . . . 374

4. Determination of the size of the exit pupil of lenses 380

Chapter XX Measurement of Reflection, Transmission and Absorption

XX-1. Measurement of reflection factor . . . . . . . 381

2. Measurement of transmission factor. . . . . . . 388

3. Measurement of absorption . . . . . . . . 390

4. Measurement of luminance factors and gloss . . . 391

\section{APPENDIX}

International co-operation in Illuminating Engineering . . . . 403

Table I - Table of solid angles . . . . . . . . . . . . 407

Table II - Values of $\cos ^{3} \alpha \cos ^{3} \beta$ for a number of values of the angles $\alpha$ and $\beta$. . . . . . . . . . . . . . . 408

Table III - Values of $\tan \beta / \cos \alpha$ for a number of values of the angles $\alpha$ and $\beta$. . . . . . . . . . . . . . . . . 410

Table IV - Units of illumination and their mutual conversion factors ............... . 412

Table V - The units of luminance and their mutual conversion factors ............... 4113

Table VI - International relative luminous efficiency of radiation for photopic vision . . . . . . . . . . . 414

Table VII - International relative luminous efficiency of radiation for scotopic vision . . . . . . . . . . 415

Light distribution and zonal luminous flux diagrams for a number of lighting fittings. . . . . . . . . . . . . . 417

Index .................. . 426 


\begin{tabular}{|c|c|c|}
\hline$\alpha$ & alpha & Plane angle, absorption factor \\
\hline$\beta$ & beta & Plane angle, luminance factor \\
\hline$\gamma$ & gamma & Plane angle \\
\hline$\delta$ & delta & Plane angle \\
\hline$\epsilon$ & epsilon & Emission factor \\
\hline$\eta$ & & Efficiency of fittings, coefficient of ultilization \\
\hline$\lambda$ & lambda & Wavelength \\
\hline$\mu$ & & Micro \\
\hline$\nu$ & $\mathrm{nu}$ & Frequency \\
\hline$\pi$ & & 3.1415 . \\
\hline$\rho$ & & Reflection factor \\
\hline$\sigma$ & sigma & $\begin{array}{l}\text { Diffusion factor, transmissive exponent of diffusing } \\
\text { media }\end{array}$ \\
\hline$\tau$ & tau & Transmission factor \\
\hline$\varphi$ & & Plane angle \\
\hline$\Psi$ & & Plane angle \\
\hline$\omega$ & omega & Solid angle \\
\hline$\Delta$ & delta & Small part. of a quantity \\
\hline$\Theta$ & theta & Half-aperture angle of light beams \\
\hline$\Sigma$ & sigma & Sum of a number of quantities \\
\hline$\Phi$ & & Luminous flux \\
\hline$a$ & & Transmissive exponent of transparent media \\
\hline$b$ & & Geographical latitude \\
\hline$c$ & & Velocity of light \\
\hline$d$ & & Distance \\
\hline$f$ & & Focal distance \\
\hline$g$ & & Optical limit distance of photometry, gloss number \\
\hline$h$ & & Height \\
\hline$i$ & & Angle of incidence \\
\hline$l$ & & Geometrical longitude, length \\
\hline$n$ & & Refractive index \\
\hline$r$ & & Angle of refraction \\
\hline$r, R$ & & Radius \\
\hline$t$ & & Time \\
\hline$u$ & & Olject distance \\
\hline$v$ & & Image distance \\
\hline$E$ & & Illumination, energy (power) \\
\hline$H$ & & Luminous emittance \\
\hline$I$ & & Luminous intensity \\
\hline$K_{\lambda}$ & & Luminous efficiency of radiation at wavelength $\lambda$ \\
\hline$L$ & & Luminance \\
\hline $\begin{array}{l}L \\
M\end{array}$ & & $\begin{array}{l}\text { Equivalent luminance } \\
\text { Mechanical equivalent of light linear magnification }\end{array}$ \\
\hline$Q$ & & $\begin{array}{l}\text { Mechanical equivalent of light, linear magnification } \\
\text { Quantity of light }\end{array}$ \\
\hline$\stackrel{s}{s}$ & & Area, density \\
\hline$T$ & & Periodic time \\
\hline$V_{\lambda}$ & & International relative luminous efficiency of radiation \\
\hline$V_{\lambda}^{\prime}$ & & $\begin{array}{l}\text { International relative luminous efficiency of radiation } \\
\text { for scotopic vision at wavelength } \lambda\end{array}$ \\
\hline
\end{tabular}




\section{LIGHT CALCULATIONS}

AND

MEASUREMENTS 